\title{
MIMO Channel Characteristics in Line-of-Sight Environments
}

\author{
Leilei Liu, Wei Hong, Nianzu Zhang, Haiming Wang and Guangqi Yang \\ State Key Lab. of Millimeter Waves, School of Information Science and Engineering, \\ Southeast University \\ China
}

\section{Introduction}

It is known that the performance of Multiple-Input-Multiple-Output (MIMO) system is highly dependent on the channel characteristics, which determined by antenna configuration and richness of scattering. In this chapter, we address the utilization issue of MIMO communication in strong line-of-sight (LOS) component propagation. It will be focused on the characteristics of the MIMO channel matrix, the channel capacity and the condition number of the matrix. Two typical scenarios will be discussed: the pure LOS environment and the LOS environment with a scatterer. Our previous researches (Liu et al., 2007 \& 2009) formed the basis of this chapter.

For the first case, the design constraint for antenna arrangement as a function of frequency and distance is discussed for the LOS MIMO communication. Then is can be seen how this constraint works and how could this constraint be weaken by smart geometrical arrangement and multi-polarization.

For the second case, the effects of scatterer on the MIMO channel characteristics in LOS environment are described. The MIMO channel matrix is expressed analytically for typical scatterer and the microstrip antennas are considered in this case. Some suggestions for practical MIMO system design will be presented in the end.

\section{MIMO channel model and channel characteristics}

\subsection{MIMO channel matrix $\mathbf{H}$}

We consider a MIMO channel model with $n_{t}$ transmit antennas and $n_{r}$ receive antennas [illustrated in Fig. 1]. The channel impulse response between the $i$ transmit antenna and the $j$ receive antenna is denoted as $h_{j, i}$. Given that the signal $x_{i}(t)$ is launched from the $i$ transmit antenna, the signal received at the $j$ receive antenna is given by

$$
y_{j}(t)=\sum_{i=1}^{n_{t}} h_{j, i}(t) * x_{i}(t)+\eta_{j}(t) \quad i=1,2, \ldots, n_{t} ; j=1,2, \ldots, n_{r}
$$

where $*$ denotes the convolution operation, $\eta_{j}(t)$ is the additive noise in the receiver. 
The channel is assumed to be frequency-flat over the band of interest, then (1) is rewritten as

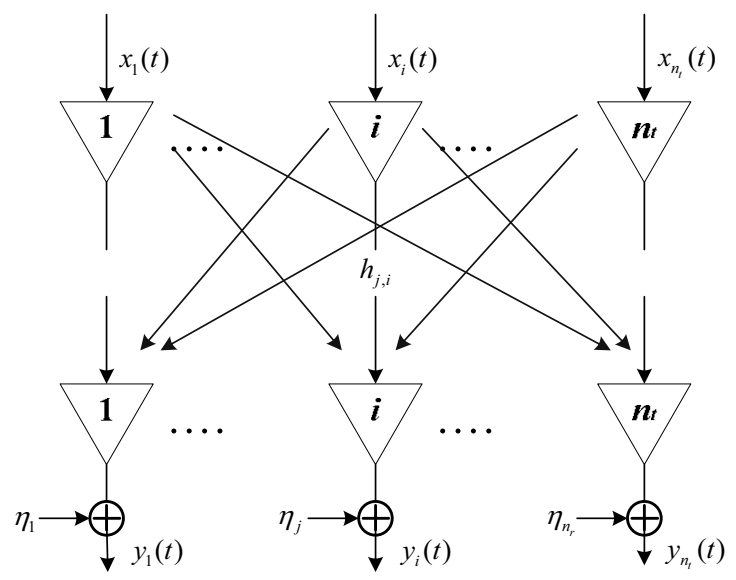

Fig. 1. MIMO channel model with $n_{t}$ transmit antennas and $n_{r}$ receive antennas

$$
y_{j}(t)=\sum_{i=1}^{n_{t}} h_{j, i}(t) x_{i}(t)+\eta_{j}(t)
$$

It can be described by matrix form

$$
\mathbf{y}(t)=\mathbf{H x}(t)+\boldsymbol{\eta}(t)
$$

where

$$
\begin{aligned}
& \mathbf{x}(t)=\left(x_{1}(t), x_{2}(t), \ldots, x_{n_{t}}(t)\right)^{\mathrm{T}} \in \mathrm{C}^{n_{t} \times 1} \\
& \mathbf{y}(t)=\left(y_{1}(t), y_{2}(t), \ldots, y_{n_{r}}(t)\right)^{\mathrm{T}} \in \mathrm{C}^{n_{r} \times 1} \\
& \boldsymbol{\eta}(t)=\left(\eta_{1}(t), \eta_{2}(t), \ldots, \eta_{n_{r}}(t)\right)^{\mathrm{T}} \in \mathrm{C}^{n_{r} \times 1}
\end{aligned}
$$

are the transmitted signal vector, the received signal vector and the zero-mean complex Gaussian noise vector respectively.

The composite MIMO channel response is given by the matrix $\mathbf{H}$ with

$$
\mathbf{H}=\left[\begin{array}{cccc}
h_{1,1} & h_{1,2} & \cdots & h_{1, n_{t}} \\
h_{2,1} & h_{2,2} & \cdots & h_{2, n_{t}} \\
\vdots & \vdots & & \vdots \\
h_{n_{r}, 1} & h_{n_{r}, 1} & \cdots & h_{n_{r}, n_{t}}
\end{array}\right] \in \mathrm{C}^{n_{r} \times n_{t}}
$$

The discrete signal model is obtained by sampling as symbol time $T_{\mathrm{s}}$

$$
\mathbf{y}(k)=\sqrt{E_{s} / n_{t}} \mathbf{H x}(k)+\boldsymbol{\eta}(k)
$$

where $E_{s}$ is the total average energy available at the transmitter over a symbol period, the normalized transmitted energy on every transmit antenna is $\sqrt{E_{s} / n_{t}}$ during a symbol period. 


\subsection{LOS component and NLOS component}

The Ricean MIMO channel model decomposes the channel into a deterministic LOS component and a stochastic NLOS (non-LOS) component for the scattered multi-path signal (Erceg et al., 2002), where the Ricean K-factor is defined as the ratio between the power of the two (Tepedelenlioglu et al., 2003), and the common path loss is moved out of the matrix $\mathbf{H}$ as being normalized.

$$
\mathbf{H}=\sqrt{\frac{K}{1+K}} \cdot \mathbf{H}_{L O S}+\sqrt{\frac{1}{1+K}} \cdot \mathbf{H}_{N L O S}
$$

Note that when $K=\infty$ the matrix becomes a pure LOS matrix and when $K=0$ it corresponds to the case of pure Rayleigh fading. As the environment we concerned is related to the microwave relay system, which is a pure LOS channel generally, our discussion will be focused on the LOS scenario, which implies $K=\infty$.

\subsection{Deterministic MIMO Channel Capacity}

Chanel capacity evaluates the performance of MIMO channels by quantifying the maximum information able to be transmitted by the propagation channel without error (Paulraj et al., 2004). We assume that the channel $\mathrm{H}$ is perfectly known to the receiver. The capacity expressed in Bit/s/Hz of the MIMO channel is given by (Telatar, 1999)

$$
C=\max _{\mathbf{R}_{\mathbf{x x}}, t r\left(\mathbf{R}_{\mathbf{x x}}\right)=n_{t}} \log _{2}\left[\operatorname{det}\left(\mathbf{I}_{n_{r}}+\frac{E_{s}}{n_{t} N_{0}} \mathbf{H R}_{\mathbf{x x}} \mathbf{H}^{\mathrm{H}}\right)\right]
$$

where $E_{s}$ is the total average energy available at the transmitter over a symbol period , $N_{0}$ is additive temporally white complex Gaussian noise. $(\cdot)^{H}$ stands for complex conjugate transpose, $\operatorname{tr}(\cdot)$ stands for trace.

$\mathbf{R}_{x x}$ is the covariance matrix of transmitted signal $x$. The maximization is performed over all possible input covariance matrices satisfying $\operatorname{tr}\left(\mathbf{R}_{x x}\right)=n_{t}$.

Given a bandwidth of $W \mathrm{~Hz}$, the maximum asymptotically error-free data rate supported by the MIMO channel is simply WCbit/s.

Assume that CSI (Channel State Information) is known only at the receiver, and then the covariance matrix should be

$$
\mathbf{R}_{\mathbf{x x}}=\mathbf{I}_{n_{t}}
$$

This implies that the signals transmitted from the individual antennas are independent and equi-powered. With (8)submitted to (7), the channel capacity is given as (Foschiniet al. , 1998)

$$
C=\log _{2}\left[\operatorname{det}\left(\mathbf{I}_{n_{r}}+\frac{E_{s}}{n_{t} N_{0}} \mathbf{H} \mathbf{H}^{\mathrm{H}}\right)\right]
$$

which may be decomposed as

$$
C=\sum_{i=1}^{m} \log _{2}\left(\mathbf{I}_{n_{r}}+\frac{E_{s}}{n_{t} N_{0}} \lambda_{i}\right)
$$


where $m=\min \left\{n_{t}, n_{r}\right\}, \lambda_{i}$ denotes the positive eigenvalues of $\mathrm{W}$, or the singular value of the matrix $\mathbf{H}$.

$$
\mathbf{W}= \begin{cases}\mathbf{H H}^{\mathrm{H}} & n_{r} \leq n_{t} \\ \mathbf{H}^{\mathrm{H}} \mathbf{H} & n_{r}>n_{t}\end{cases}
$$

Equation (10) expresses the spectral efficiency of the MIMO channel as the sum of the capacities of $m$ SISO channels with corresponding channel gains $\sqrt{\lambda_{i}}(i=1,2, \ldots m)$ and transmit energy $E_{s} / n_{t}$ (Paulraj et al., 2004).

\subsection{Condition number of the channel matrix}

The condition number of the channel matrix is the second important characteristic parameter to evaluate the environmental modelling impact on MIMO propagation. It is known that low-rank matrix brings correlations between MIMO channels and hence is incapable of supporting multiple parallel data streams. Since a channel matrix of full rank but with a large condition number will still bring high symbol error rate, condition number is preferred to rank as the criterion.

The condition number is defined as the ratio of the maximum and minimum singular value of the matrix $\mathbf{H}$.

$$
\operatorname{cond}(\mathbf{H})=\frac{\lambda_{\max }(\mathbf{H})}{\lambda_{\min }(\mathbf{H})}
$$

The closer the condition number gets to one, the better MIMO channel quality is achieved. As a multiplication factor in the process of channel estimation, small condition number decreases the error probability in the receiver.

\section{MIMO technique utilized in LOS propagation}

As discussed above, the high speed data transmission promised by the MIMO technique is highly dependent on the wireless MIMO channel characteristics. The channel characteristics are determined by antenna configuration and richness of scattering. In a pure LOS component propagation, low-rank channel matrix is caused by deficiency of scattering (Hansen et al., 2004).

Low-rank matrix brings correlations between MIMO channels and hence is incapable of supporting multiple parallel data streams. But some propagation environments, such as microwave relay in long range communication and WLAN system in short range communication, are almost a pure LOS propagation without multipath environment. However, by proper design of the antenna configuration, the pure LOS channel matrix could also be made high rank. It is interesting to investigate how to make MIMO technique utilized in LOS propagation.

\subsection{The design constraint}

We firstly consider a symmetrical $4 \times 4$ MIMO scheme with narrow beam antennas. The practical geometric approach is illustrated in Fig. 2, this geometrical arrangement can extend the antenna spacing and hence reduce the impact of MIMO channel correlation. On each 
side, the four antennas numbered clockwise are distributed on the corners of a square with the antenna spacing $d$. R represents the distance between the transmitter and the receiver.

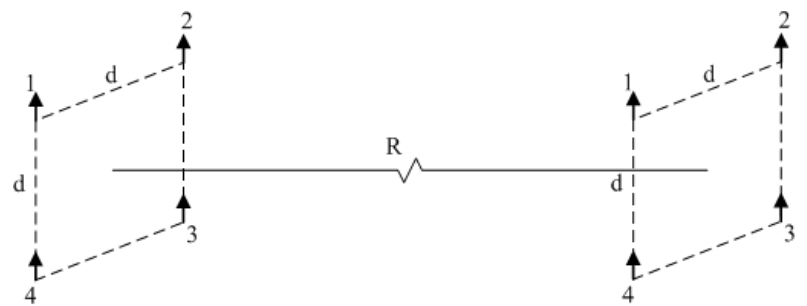

Fig. 2. Arrangement of $4 \mathrm{Rx}$ and $4 \mathrm{Tx}$ antennas model

We assume the distance $R$ is much larger than the antenna spacing $d$. This assumption results in a plane wave from the transmitter to the receiver. In addition, the effect of path loss differences among antennas can be ignored, only the phase differences will be considered.

From the geometrical antenna arrangement, we have the different path lengths $r_{m, n}$ from transmitting antenna $n$ to receive antenna $m$ :

$$
\begin{gathered}
r_{1,1}=R \\
r_{2,1}=r_{4,1}=\sqrt{R^{2}+d^{2}} \approx R+d^{2} /(2 R) \\
r_{3,1}=\sqrt{R^{2}+2 d^{2}} \approx R+d^{2} / R, \ldots
\end{gathered}
$$

All the approximations above are made use of first order Taylor series expansion, which becomes applicable when the distance is much larger than antenna spacing.

Denoting the received vector from transmitting antenna $n$ as

$$
\mathbf{h}_{n}=\left[\exp \left(-j \frac{2 \pi}{\lambda} r_{1, n}\right), \ldots, \exp \left(-j \frac{2 \pi}{\lambda} r_{4, n}\right)\right]^{T}, n=1 \ldots 4
$$

where $\lambda$ is the wavelength and $(\cdot)^{T}$ denotes the vector transpose. Thus the channel matrix is given as

$$
\mathbf{H}=\left[\mathbf{h}_{1}, \mathbf{h}_{2}, \mathbf{h}_{3}, \mathbf{h}_{4}\right]
$$

The best situation for the channel matrix is that its condition number (11) equals to one. It is satisfied when $\mathbf{H}$ is the full orthogonality matrix which means all the columns (or rows) are orthogonal.

Orthogonality between different columns in (13) is obtained if the inner product of two received vectors from the adjacent transmitting antennas equals to zero:

$$
<h_{k}, h_{k+1}>=2 \exp \left(-j \frac{2 \pi}{\lambda}\left(2 R+\frac{d^{2}}{2 R}\right)\right)\left[1+\exp \left(-j \frac{2 \pi}{\lambda} \cdot \frac{d^{2}}{R}\right)\right]=0
$$

which results in

$$
d^{2}=\frac{\lambda R}{2}(2 k+1) \quad k=0,1 \ldots
$$


To get practical values of $d$, we choose $k=0$ to update (15). The optimal design constraint therefore becomes

$$
d^{2}=\frac{\lambda R}{2}
$$

From (16) we can see the optimal antenna spacing is a function of carrier frequency and propagation distance as well as the geometrical arrangement. As $\lambda=c / f$ shows, higher frequency results in smaller antenna spacing requirement, but longer distance increases it. Here $c$ denotes the velocity of light. According to the deriving process above, it is obvious that when constraint (16) is satisfied, the channel matrix $\mathbf{H}$ in (16) is a full-rank matrix. This optimal design constraint is also determined by the antenna array arrangement, since different path lengths $r_{m, n}$ gives different channel matrix.

The derivation foundation of (16) is the condition number of $\mathbf{H}$ in (11) equals to one, which also satisfies all singular values of $\mathrm{H}$ are equal, that is

$$
\lambda_{1}(\mathbf{H})=\cdots=\lambda_{4}(\mathbf{H})
$$

From (17) and the method of Lagrange multipliers, the highest channel capacity in (10) is obtained (Bohagen et al. 2005). Therefore, maximum capacity and best condition number agree well.

The relation between the condition number and the capacity in pure LOS propagation is depicted in Fig. 3. The capacity is in linear inverse proportion to condition number, i.e., the closer the condition number is to one, higher the capacity is. It achieves the maximum capacity of $17.6 \mathrm{bit} / \mathrm{s} / \mathrm{Hz}$ at $3 \mathrm{~km}$ transmission distance with $30 \mathrm{GHz}$ frequency and $20 \mathrm{~dB}$ SNR (Signal to Noise Ratio).

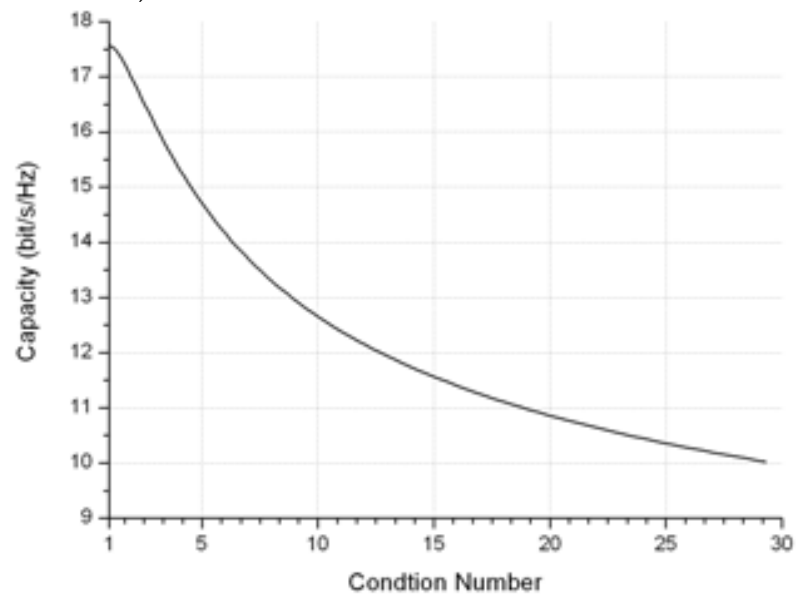

Fig. 3. Capacity as a function of condition number in pure LOS propagation, for the case that SNR $20 \mathrm{~dB}$, optimal frequency $30 \mathrm{GHz}$, and optimal transmit distance $3 \mathrm{~km}$. 


\subsection{MIMO channel characteristics analysis and suggestions}

Antenna spacing larger than half wavelength is usually required for achieving uncorrelated subchannels in dense scattering environment (Foschini, 1996). The design constraint in (16) shows half wavelength is no longer enough for pure LOS propagation when distance between transmitter and receiver is large. In the following, we will discuss how to construct a feasible LOS MIMO channel in accordance with this design constraint. It is also interesting to explore what is the acceptable situation and how it affects the practical design.

Based on the $4 \times 4$ MIMO ray tracing model above, the relation between condition number and antenna spacing is investigated and shown in Fig. 4. It confirms that larger distance requires larger antenna spacing while higher frequency requires smaller antenna spacing. The optimal condition number can be achieved at many points because of the periodicity of traveling wave phase.

The design constraint in (16) obtains the optimal channel quality, but the large antenna spacing is difficult to achieve in practice. However, practically, the condition number around 10 is allowed from the view of link quality. For example, if carrier frequency and transmission distance are $30 \mathrm{GHz}$ and $2 \mathrm{~km}$ respectively, instead of $3.2 \mathrm{~m}$ antenna spacing for the best case, $2 \mathrm{~m}$ antenna spacing also performs well as condition number equals to 10 .

It is noted that the antenna spacing is fairly large, but potentially acceptable for microwave relay or mobile telephone towers. In addition, NLOS elements also exist in actual situation, such as weak scattering elements, rain event, etc. These causes will increase the independence of MIMO channels and therefore improve the condition number.

Some position errors will exist in practical setting, and Fig. 5 investigates how sensitive the performance of channel matrix is to the distance between two relay stations, with different or frequency $30 \mathrm{GHz}$ or $40 \mathrm{GHz}$, and different antenna spacing $2 \mathrm{~m}$ or $4 \mathrm{~m}$ respectively. It shows that these four scenarios have the same degradation rate of channel quality with the

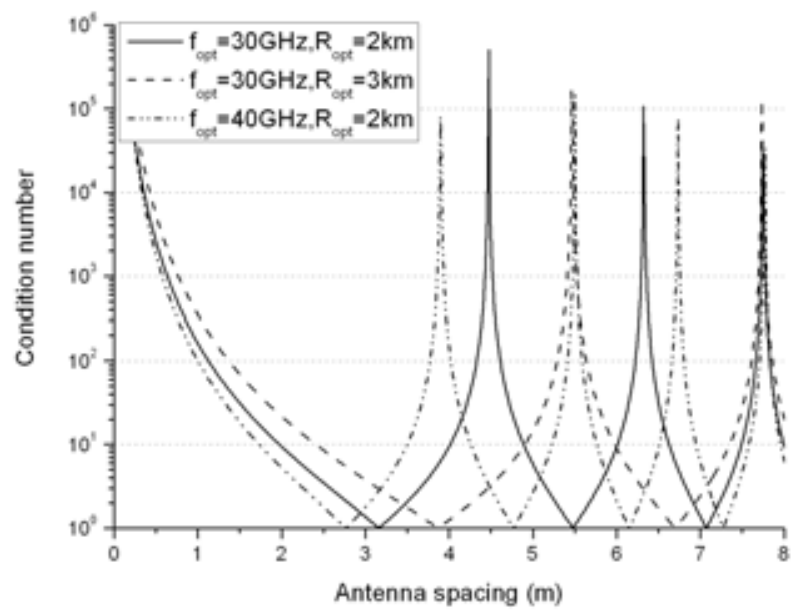

Fig. 4. Antenna spacing deviation impact on condition number with fixed frequency $(30 \mathrm{GHz}, 40 \mathrm{GHz})$ and fixed distance $(2 \mathrm{~km}, 3 \mathrm{~km})$. Condition number below $10 \mathrm{can}$ be accepted in practice. 


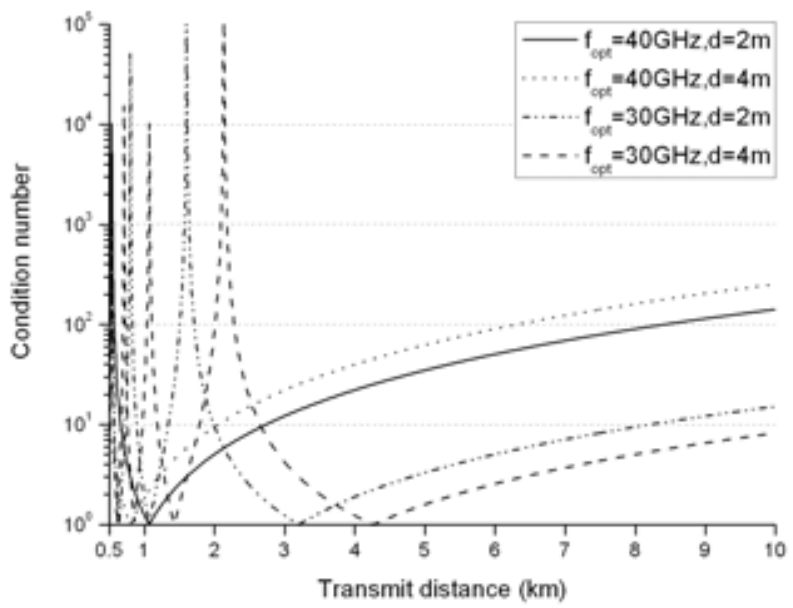

Fig. 5. Transmit distance deviation impact on condition number with fixed frequency $(30 \mathrm{GHz}, 40 \mathrm{GHz})$ and optimal antenna spacing $(2 \mathrm{~m}, 4 \mathrm{~m}) .1000 \mathrm{~m}$ location deviation yields slight performance degradation.

distance offsets. This figure also indicates that even 1000 meters location deviation yields slight performance degradation.

\subsection{Effects of multi-polarization}

As the design constraint shows in (16), considerable antenna spacing is needed to introduce phase differences among antennas when operating MIMO system in LOS environment. To increase the independence among MIMO channels, multi-polarized antennas can be applied. Using the same geometry depicted in Fig. 2, we assume that $\alpha_{i}(i=1,2,3,4)$ denotes the offset angle of the polarization of $i$ th transmitting antenna with respect to vertical polarization, while $\beta_{j}(j=1,2,3,4)$ denotes the offset angle of the polarization of $j$ th receiving antenna. For simplicity, we neglect the effect of cross polarization. Then the channel matrix (12) in multi-polarized LOS MIMO scenario is updated to

$$
\mathbf{h}_{n}=\left[\cos \left(\beta_{1}-\alpha_{n}\right) \cdot \exp \left(-j \frac{2 \pi}{\lambda} r_{1, n}\right), \ldots, \cos \left(\beta_{4}-\alpha_{n}\right) \cdot \exp \left(-j \frac{2 \pi}{\lambda} r_{4, n}\right)\right]^{T}, \quad n=1, \ldots, 4
$$

where $\cos \left(\beta_{j}-\alpha_{i}\right)$ is the square root of normalized signal power on $j$ th receiving antenna relative to $i$ th transmitting antenna. With regard to this new channel matrix, we will see the improvements of channel matrix characteristics brought by multi-polarization.

Fig. 6 illustrates how the multi-polarization impacts on the MIMO channel characteristic. Three typical polarized cases are plotted compared with the uni-polarized case. By searching all the values of polarization degree in $\left[0^{\circ}, 90^{\circ}\right]$, some points can be concluded: the use of multi-polarized antennas is an effective way to decrease the antenna spacing. 


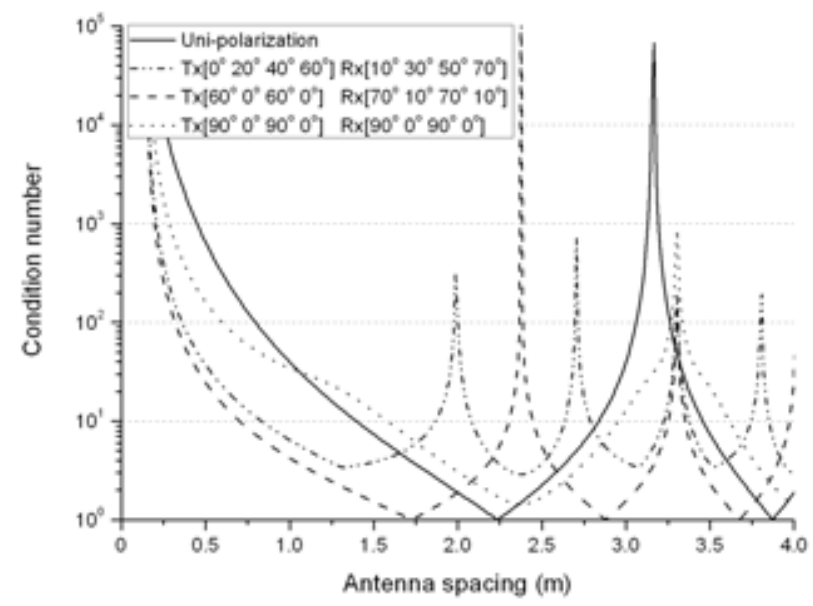

Fig. 6. Condition number as a function of antenna spacing with three polarized cases compared to the uni-polarized case. Degree of polarized antennas on transmitter side (Tx) and receiver side (Rx) follows: Case1: $\mathrm{Tx} 0^{\circ}, 20^{\circ}, 40^{\circ}, 60^{\circ}, \mathrm{Rx} 10^{\circ}, 30^{\circ}, 50^{\circ}, 70^{\circ}$; Case2: Tx $60^{\circ}, 0^{\circ}$, $60^{\circ}, 0^{\circ}, \operatorname{Rx} 70^{\circ}, 10^{\circ}, 70^{\circ}, 10^{\circ}$; Case 3 : $\mathrm{T} \times 90^{\circ}, 0^{\circ}, 90^{\circ}, 0^{\circ}, \mathrm{R} \times 90^{\circ}, 0^{\circ}, 90^{\circ}, 0^{\circ}$. The use of multi-polarization appears as a space- and cost-effective alternative.

For instance, case 2 saves $0.8 \mathrm{~m}$ antenna spacing to achieve condition number 10 relative to the uni-polarized case. Moreover, dual-polarization on each side leads to better channel matrix characteristic than four-polarization. Furthermore, the minimal antenna spacing we get is the orthogonal polarization $0^{\circ} / 90^{\circ}$ on each side. But this is not the best choice for system performance, because the improvement of channel quality is based on sacrificing the transmitting power and the receiving diversity gain.

\section{Effects of scatterer on the LOS MIMO channel}

As we discuss above, the implement of MIMO technique to pure LOS propagation enviroment is restricted by a constraint which is a function of antenna arrangement, frequency and transmission distance. In actual outdoor radio channels, the existence of scattering will improve the MIMO channel performance effectively (Gesbert et al., 2002). Start from the electromagnetic knowleges, we will give the theoretical explanation on how the channel performance improved and how much it will be improved by a typical scatter.

\subsection{A 2D MIMO channel model in outdoor propagation}

We focused on the outdoor LOS environment but with a scatterer. It is an abstract model for the propagation enviroment of microwave relay or mobile telephone towers. Analytical method will be adopted in this channel model combining the electromagnetic theory and antenna theory. 


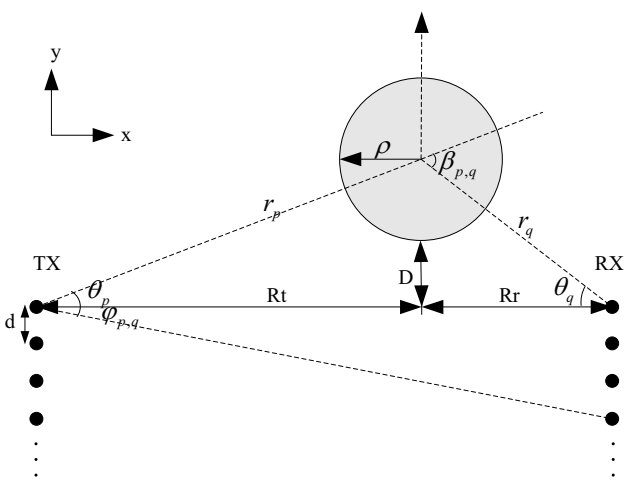

Fig. 7. A 2D MIMO channel model in outdoor propagation

A 2D MIMO channel model in outdoor LOS propagation is shown Fig. 7. Combining with the practical applications, microstrip patch array antennas are used in this model. Every rectangular patch antenna on each side is arranged along z-axis. To simplify the MIMO system is projected to $x-y$ plane, it has $P$ transmitter and $Q$ receiver. The propagation is considered as a LOS situation, a cylindrical scatter is on the side of the direct path. The cylinder is the simplified model of the actual architecture in outdoor environments. Only transverse magnetic wave (vertical polarization) is considered in this electromagnetic scattering problem.

\subsection{Radiation patterns of microstrip antennas}

The geometry for far-field pattern of rectangular microstrip patch is shown in Fig. 8. The farfield radiation pattern of such a rectangular microstrip patch operation in the $\mathrm{TM}_{10}$ mode is broad in both the $\mathrm{E}$ and $\mathrm{H}$ planes. The pattern of a patch over a large ground plane may be calculated by modelling the radiator as two parallel uniform magnetic line sources of length $a$, separated by distance $b$. If the slot voltage across either radiating edge is taken as $V_{0}$, the calculated fields are (Carver et. al, 1981)

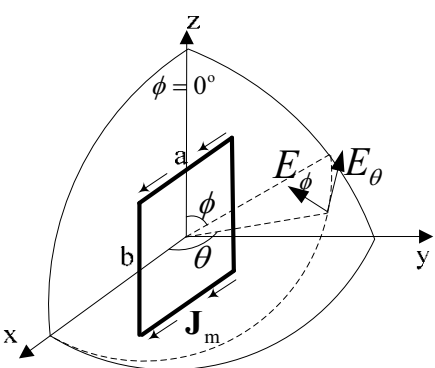

Fig. 8. Geometry for far-field pattern of rectangular microstrip patch 


$$
\begin{aligned}
E_{\theta} & =\frac{j V_{0} k_{0} a e^{-j k_{0} r}}{\pi r}[\cos (k h \cos \theta)] \cdot\left[\frac{\sin \left[k_{0} \frac{a}{2} \sin \theta \sin \phi\right]}{k_{0} \frac{a}{2} \sin \theta \sin \phi}\right] \\
& \cdot\left[\cos \left(k_{0} \frac{b}{2} \sin \theta \cos \phi\right)\right] \cos \phi, \quad\left(0 \leq \theta \leq \frac{\pi}{2}\right) \\
E_{\phi}= & \frac{j V_{0} k_{0} a e^{-j k_{0} r}}{\pi r}[\cos (k h \cos \theta)] \cdot\left[\frac{\sin \left[k_{0} \frac{a}{2} \sin \theta \sin \phi\right]}{k_{0} \frac{a}{2} \sin \theta \sin \phi}\right] \\
& \cdot\left[\cos \left(k_{0} \frac{b}{2} \sin \theta \cos \phi\right)\right] \cos \theta \sin \phi, \quad\left(0 \leq \theta \leq \frac{\pi}{2}\right),
\end{aligned}
$$

where $\mathrm{h}$ is the substrate thickness, $k=k_{0} \sqrt{\varepsilon_{r}}, k_{0}$ is the wave number in vacuum, $\varepsilon_{r}$ is the dielectric constant, $r$ is the radiation distance.

\subsection{Cylindrical scattering}

To obtain the analytical expression, we suppose the cylindrical scatter in Fig. 7 is a conducting cylinder with the radius $\rho$. The plane wave incident upon this cylinder is considered since the propagation distance from the transmitter to the cylinder is long enough.

Take the incident wave to be z-polarized, that is (Harrington, 2001)

$$
E_{z}^{i}=E_{0} e^{-j k r \cos \beta}
$$

where $E_{0}$ is the far-field from transmitter to cylinder, $r$ is the propagation distance and $\beta$ is the scattering angle in Fig. 7.

Using the wave transformation, we can express the incident field as

$$
E_{z}^{i}=E_{0} \sum_{n=-\infty}^{\infty} j^{-n} J_{n}(k r) e^{j n \beta}
$$

where $J_{n}$ is the first kind Bessel function.

The total field with the conducting cylinder present is the sum of the incident and scattered fields, that is

$$
E_{z}=E_{z}^{i}+E_{z}^{s}
$$

To presnet outward-traveling waves, the scattered field must be of the form

$$
E_{z}^{s}=E_{0} \sum_{n=-\infty}^{\infty} j^{-n} a_{n} H_{n}^{(2)}(k r) e^{j n \beta}
$$

where $H_{n}^{(2)}$ is the second kind Hankel function.

Hence the total field is

$$
E_{z}=E_{0} \sum_{n=-\infty}^{\infty} j^{-n}\left[J_{n}(k r)+a_{n} H_{n}^{(2)}(k r)\right] e^{j n \beta}
$$


At the cylinder the boundary condition $E_{z}=0$ at $r=\rho$ must be met. It is evident from the above equation that this condition is met if

$$
a_{n}=\frac{-J_{n}(k \rho)}{H_{n}^{(2)}(k \rho)}
$$

Which completes the solution.

\subsection{Analytical mathematical expression of channel matrix $\mathbf{H}$}

According to 2D model in Fig. 7, $\phi=90^{\circ}$ is adopted in the far-field radiation pattern of patch antenna. Thus, $E_{\theta}=0$, and the incident wave from each microstrip antenna of the transmitter to the cylinder is

$$
E_{z}^{i}=\frac{j V_{0} k_{0} a e^{-j k_{0} r}}{\pi r}[\cos (k h \cos \theta)]\left[\frac{\sin \left[k_{0} \frac{a}{2} \sin \theta\right]}{k_{0} \frac{a}{2} \sin \theta}\right] \cos \theta, \quad\left(0 \leq \theta \leq \frac{\pi}{2}\right)
$$

In accordance with the Geometric Relationship between the cylinder and each antenna shown in Fig. 7, the scattered field from the $p$ antenna in transmitter to the $q$ antenna in receiver affected by the cylinder is calculated by (24) and (27)

$$
\begin{aligned}
E_{p, q}^{s}= & \frac{j V_{0} k_{0} a}{\pi r_{p}} \exp \left(-j k_{0} r_{p}\right) \cdot\left[\cos \left(k h \cos \theta_{p}\right)\right] \cdot\left[\frac{\sin \left[k_{0}(a / 2) \sin \theta_{p}\right]}{k_{0}(a / 2) \sin \theta_{p}}\right] \cdot \cos \theta_{p} \\
& \cdot \sum_{n=-N}^{N} j^{-n} \frac{-J_{n}\left(k_{0} \rho\right)}{H_{n}^{(2)}\left(k_{0} \rho\right)} H_{n}^{(2)}\left(k_{0} r_{q}\right) \exp \left(j n \beta_{p, q}\right) \quad p=1 \ldots P, q=1 \ldots Q
\end{aligned}
$$

where $r_{p}$ is the distance from the $p$ transmit antenna to the cylinder, $r_{q}$ is the distance from the cylinder to the $q$ receive antenna. $\theta_{p}$ is the angle between the LOS path and the ray path from the $p$ transmit antenna to the cylinder, $\theta_{q}$ is the angle between the LOS path and the ray path from the cylinder to the $q$ receive antenna. $\beta_{p, q}$ is the scattering angle in Fig. 7 , which $\beta_{p, q}=\theta_{p}+\theta_{q}$. The value of $N$ order is determined by the convergence of the Bessel function and the Hankel fuction.

The incident wave at the receiver from the LOS path is

$$
\begin{aligned}
E_{p, q}^{i}= & \frac{j V_{0} k_{0} a}{\pi R_{p, q}} \exp \left(-j k_{0} R_{p, q}\right) \cdot\left[\cos \left(k h \cos \varphi_{p, q}\right)\right] \\
& \cdot\left[\frac{\sin \left[k_{0}(a / 2) \sin \varphi_{p, q}\right]}{k_{0}(a / 2) \sin \varphi_{p, q}}\right] \cos \varphi_{p, q}, \quad p=1 \ldots P, q=1 \ldots Q
\end{aligned}
$$

where $R_{p, q}$ and $\varphi_{p, q}$ are the distance and the angle between the $p$ transmit antenna and the $q$ receive antenna respectively.

The antenna directivity of the receiving antenna is given by the rectangle microstrip antenna pattern 


$$
D(\theta)=[\cos (k h \cos \theta)] \cdot\left[\frac{\sin \left[k_{0}(a / 2) \sin \theta\right]}{k_{0}(a / 2) \sin \theta}\right] \cdot \cos \theta
$$

The total field is the sum of the incident and scattered fields, that is

$$
\begin{aligned}
E_{p, q} & =E_{p, q}^{i} \cdot D\left(\varphi_{p q}\right)+E_{p, q}^{s} \cdot D\left(\theta_{q}\right) \\
= & \frac{j V_{0} k_{0} a}{\pi R_{p, q}} \exp \left(-j k_{0} R_{p, q}\right)\left[\cos \left(k h \cos \varphi_{p, q}\right)\right]^{2}\left[\frac{\sin \left[k_{0}(a / 2) \sin \varphi_{p, q}\right]}{k_{0}(a / 2) \sin \varphi_{p, q}}\right]^{2}\left(\cos \varphi_{p, q}\right)^{2} \\
& +\frac{j V_{0} k_{0} a}{\pi r_{p}} \exp \left(-j k_{0} r_{p}\right)\left[\cos \left(k h \cos \theta_{p}\right)\right]\left[\frac{\sin \left[k_{0}(a / 2) \sin \theta_{p}\right]}{k_{0}(a / 2) \sin \theta_{p}}\right] \cos \theta_{p} \cos \theta_{q} \\
& \cdot \sum_{n=-N}^{N} j^{-n} \frac{-J_{n}\left(k_{0} \rho\right)}{H_{n}^{(2)}\left(k_{0} \rho\right)} H_{n}^{(2)}\left(k_{0} r_{q}\right) \exp \left(j n \beta_{p, q}\right)\left[\cos \left(k h \cos \theta_{q}\right)\right]\left[\frac{\sin \left[k_{0}(a / 2) \sin \theta_{q}\right]}{k_{0}(a / 2) \sin \theta_{q}}\right]
\end{aligned}
$$

This can be also considered as the sum energy of the LOS element and the NLOS element at the receiver. This electromagnetic interpretation agrees well with the Ricean model in (6).

Define $E_{p}^{T X}$ as the transmitted field, thus the channel matrix element follows

$$
h_{p, q}=E_{p, q} / E_{p}^{T X}
$$

Hence, the MIMO channel matrix is composed

$$
\mathbf{H}=\left[\begin{array}{ccc}
h_{1,1} & \cdots & h_{1, P} \\
\cdots & \cdots & \cdots \\
h_{1, Q} & \cdots & h_{P, Q}
\end{array}\right] \in C^{P \times Q}
$$

\subsection{Numerical Evaluation}

Our simulation is based on a $4 \times 4$ MIMO system with working frequency at $3 \mathrm{GHz}$. The antennas are excited by voltage $1 \mathrm{~V}$. The dielectric constant of the microstrip antennas substrate is 2.5 , and its thickness is $0.03 \lambda$ which determined by the working wavelength. For a matched antenna, the size could be referenced to (Bahl, et al., 1982).

The simulation parameters are initialized as follows: the spacing between antenna elements is $d=0.4 \mathrm{~m}$; the radius of cylinder is $\rho=50 \mathrm{~m}$ as the actual size of buildings; the propagation distance between transmitter and the receiver is $R=1 \mathrm{~km}$; the projected distance from the cylinder to the transmitter and to the receiver are $R t=800 \mathrm{~m}$ and $R r=200 \mathrm{~m}$ respectively; the distance between the cylinder to the LOS path is $D$.

We mentioned above the order $N$ in the scattering field expression (28) is determined by the convergence of Bessel function and Hankel function. Because the practical scatter is relatively big, large $N$ is needed. Hence, we need to investigate the convergence of these functions first, in order to reduce the calculation complexity.

We redefine the determinative part in (28) as

$$
f(n)=\sum_{n=-N}^{N} j^{-n} \frac{-J_{n}\left(k_{0} \rho\right)}{H_{n}^{(2)}\left(k_{0} \rho\right)} H_{n}^{(2)}\left(k_{0} r_{q}\right) \exp \left(j n \beta_{p, q}\right)
$$




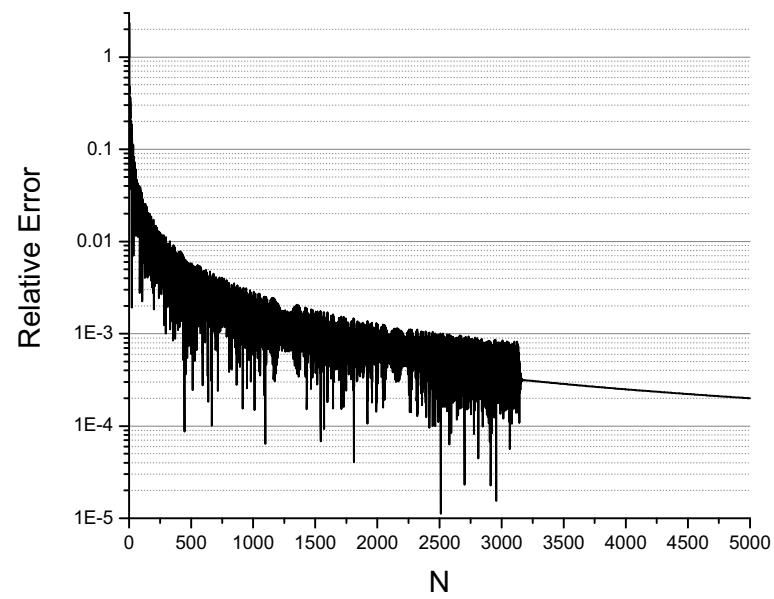

Fig. 9. The relative error function $\varepsilon(n)$, at $f=3 \mathrm{GHz}, \rho=50 \mathrm{~m}, R r=200 \mathrm{~m}$, and $D=100 \mathrm{~m}$

the relative error function is

$$
\varepsilon(n)=\frac{|f(n+1)-f(n)|}{|f(n)|}
$$

The relation between the error function $\varepsilon(n)$ and the order $N$ is shown in Fig. 9. This curve corresponds to the MIMO system with working frequency $f=3 \mathrm{GHz}$, and geometric parameters $\rho=50 \mathrm{~m}, R r=200 \mathrm{~m}$ and $D=100 \mathrm{~m}$. It shows that when $N$ is larger than 2500 , it has $\varepsilon<10^{-3}$. The $\varepsilon(n)$ curve starts smoothly when $N$ is larger than 3170 . The simulation shows that the value of $N$ makes a strong effect on the accuracy. With accordance to (34), a higher frequency, a larger scatter or a longer propagation distance needs a larger $N$ to meet the same accuracy.

Suppose the transmitted power doesn't depend on the system frequency and propagation distance. The SNR is defined as a variable which depend on the system parameters and the actual propagation environment. Set $\mathrm{SNR}_{0}=10 \mathrm{~dB}$ at $3 \mathrm{GHz}$ system frequency and $2 \mathrm{~km}$ propagation distance. Then the SNR can be calculated by the transmission loss $L_{b f}$ in free space:

$$
S N R=S N R_{0}+\left(L_{b f 0}-L_{b f}\right)
$$

where $L_{b f}=20 \lg (4 \pi R / \lambda)(\mathrm{dB})$ 


\subsection{MIMO channel characteristics analysis and suggestions}

Fig. 10 shows the effects of difference cylinder size on the MIMO channel performance. The cylinder distance to the LOS path steps by $10 \mathrm{~m}$ in the simulation. Compared with the pure LOS case, the scattering in MIMO propagation improves the channel performance significantly. The larger cylinder, the higher channel capacity achieves. If the cylinder radius is $100 \mathrm{~m}$, the channel capacity improves more than $2 \mathrm{bps} / \mathrm{Hz}$. Fig. $10(\mathrm{~b})$ shows the correlation of the MIMO sub-channels from the condition number of channel matrix. When the distance from cylinder to LOS path is smaller than $200 \mathrm{~m}$, the condition number reduces from 1E7 to $1 \mathrm{E} 5$ because of the cylindrical scattering.

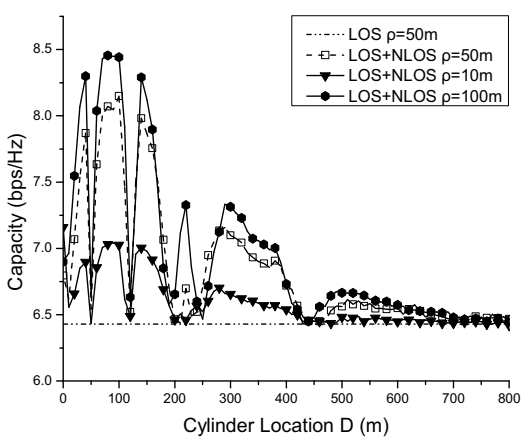

(a)

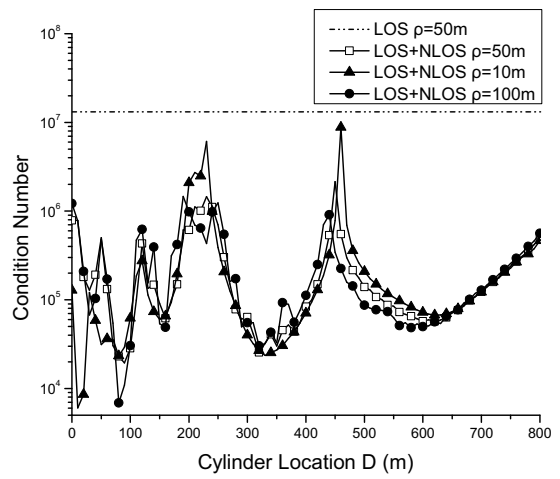

(b)

Fig. 10. The channel capacity (a) and the condition number (b) vary with the cylinder location for the different cylinder size

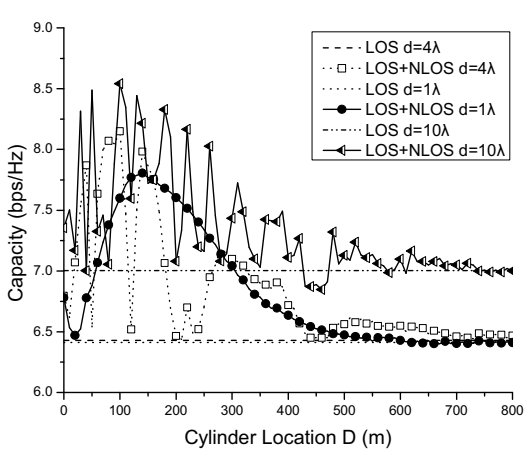

(a)

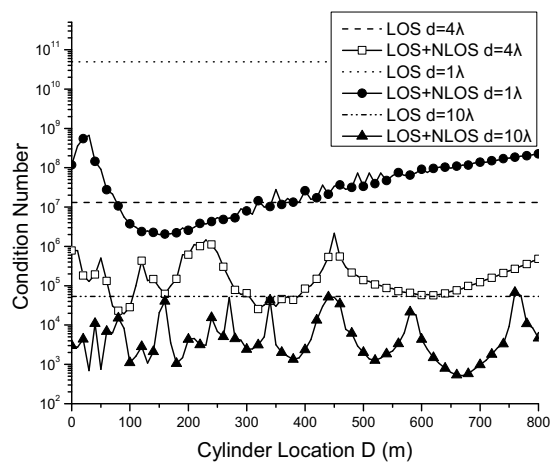

(b)

Fig. 11. The channel capacity (a) and the condition number (b) vary with the cylinder location for the different antenna spacing 
From the comparison about Fig. 10(a) and Fig. 10(b), the capacity is inversely related to the condition number. Namely, the improvement on the capacity by the cylindrical scattering is the same as the condition number. Increase with the distance from the cylinder to the LOS path, the channel performance improves gradually. It behaves as the slow fading channel. This improvement is comparatively small when the cylinder is farther away than $600 \mathrm{~m}$. However, big fluctuation appears when the cylinder is near LOS path $(D<200 \mathrm{~m})$. This is because of the fast fading caused by the superposition of the random phases in different multipath.

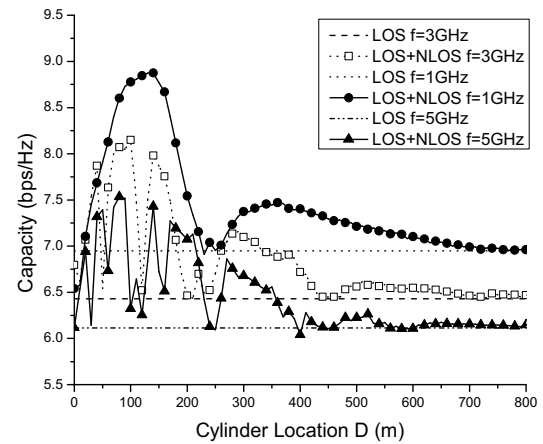

(a)

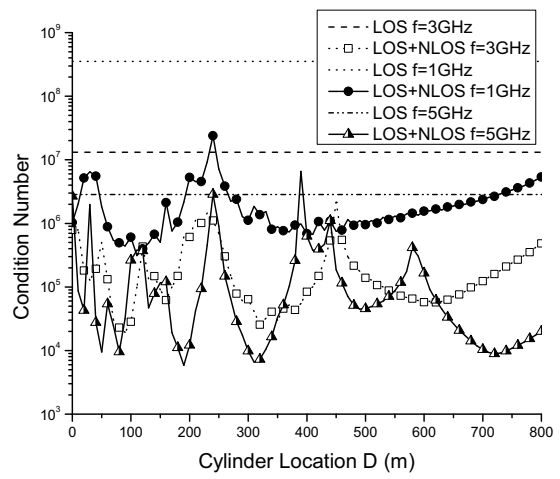

(b)

Fig. 12. The channel capacity (a) and the condition number (b) vary with the cylinder location for the different frequency

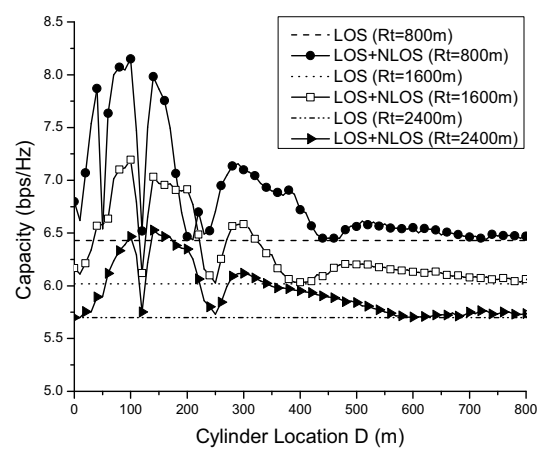

(a)

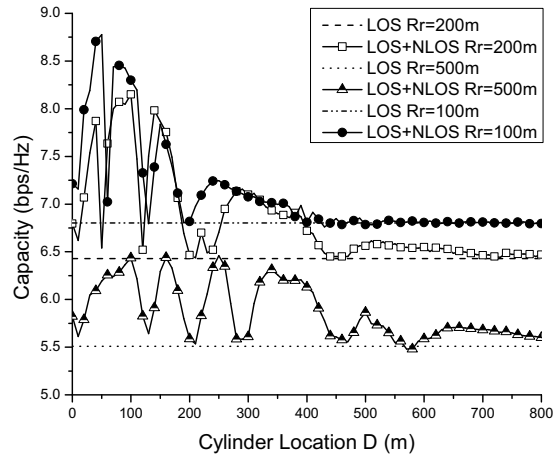

(b)

Fig. 13. The channel capacity (a) and the condition number (b) vary with the cylinder location for the different distance Rt

Fig. 11 shows that the antenna spacing increase, the correlation the subchannels becomes lower. Hence, larger capacity and smaller condition number are obtained. Besides, the 
channel performance is improved significantly. In addition, larger antenna spacing leads to bigger fluctuation of the capacity curves and the condition number curves.

Fig. 12 shows the different frequencies effect on the channel performance. It demonstrates that lower frequency results to less path loss and less sensitivity of fast fading. Namely, high frequency lets the curves fluctuate greatly.

Fig. 13 shows the different distances to the cylinder effect on the channel capacity. $R t$ is the projective distance from the transmitter to the cylinder, and $R r$ is the projective distance from the cylinder to the receiver. The further cylinder away from the antennas, the less improvement by the scattering could be achieved. Consequently, the channel capacity becomes lower. In Fig. 13(b) the slope of the curve $R r=100 \mathrm{~m}$ is larger than the $R r=500 \mathrm{~m}$ case. This is because of the received antenna orientation. With the increase distance, the angle among the received antenna and the cylinder becomes larger, which results in less received energy in former case.

There are several singular points in the curves from Fig. 10 to Fig. 13. At these points, few improvements of channel performance could be achieved. The reason for this is that the cylinder scattering causes plenty of multipath element, and the phases of these multipath superpose randomly. In the net-establishing of the base station, if the main scatters are known, the singular points could be avoided by simulated prediction.

\section{Conclusion}

In this chapter, we discussed the MIMO channel performance in the LOS environment, classified into two cases: the pure LOS propagation and the LOS propagation with a typical scatter.

We first deduced a useful constraint for the MIMO system applied in the pure LOS propagation. This constraint is a function of the antenna setup, the frequency and the transmission distance. Then we give the analytical mathematical expression of the MIMO channel matrix for the second environment case. From the electromagnetic explanation, we know how this scatter effects on the MIMO channel.

The MIMO channel capacity and the condition number of the matrix were investigated. We discussed how to construct such a LOS MIMO channel and how much a typical scatter could improve on the channel performance.

\section{References}

Bahl, I.; Bhartia, P. \& Stuchly, S. (1982). Design of microstrip antennas covered with a dielectric layer. IEEE Trans. Antennas and Propag. Vol. 30, No. 2, pp. 314-318

Bohagen, F.; Orten, P. \& Oien, G.E. (2005). Construction and capacity analysis of high-rank line-of-sight MIMO channels, Proceedings of Wireless Communications and Networking Conference, Vol. 1, pp. 432-437

Carver, K.; Mink, J. (1981). Microstrip antenna technology. IEEE Trans. Antennas and Propag. Vol. 29, No. 1, pp. 2-24

Erceg, V.; Soma, P.; Baum, D. S. \& Paulraj, A. J. (2002). Capacity obtained from multipleinput multiple-output channel measurements in fixed wireless environments at 2.5 $\mathrm{GHz}$, Proceedings of IEEE International Conference on Communications, Vol.1, pp. 396-400, April 2002 
Foschini, G. J. (1996). Layered space-time architecture for wireless communication in a fading environment when using multi-element antennas, Bell Labs Tech. J., Vol. 1, No. 2, pp. 41-59.

Foschini, G. J. \& Gans, M. J. (1998). On limits of wireless communications in a fading environment when using multiple antennas, Wireless Pers. Commun., Vol. 6, No. 3, pp. 311-335

Gesbert, D.; Bolcskei, H.; et al. (2002). Outdoor MIMO wireless channels: models and performance prediction. IEEE Trans. Commun., Vol. 50, No. 12, pp. 1926-1934.

Hansen, J. \& Bölcskei, H. (2004). A geometrical investigation of the rank-1 Ricean MIMO channel at high SNR, Proceeding of International Symposium on Information Theory. pp. 64

Harrington, R. F. (2001). Time-harmonic Electromagnetic Fields. IEEE Press Series on Electromagnetic Wave Theory.

Liu, L.; Hong W.; et al. (2007). Characterization of Line-of-Sight MIMO Channel for Fixed Wireless Communications, IEEE Antenna and Wireless Propagation Letters,Vol.6, pp. 36-39

Liu, L.; Hong W.; (2009). Investigations on the Effects of Scatterers on the MIMO Channel Characteristics in LOS Environment, Journal on Communications,Vol. 30, No.2, pp: 65-70

Paulraj, A.J.; GORE, D.A.; NABAR, R.U. \& BOLCSKEI, H. (2004). An overview of MIMO communications - a key to gigabit wireless, Proceedings of the IEEE,Vol. 92, No. 2, pp: $198-218$

Telatar, E. (1999). Capacity of Multi-Antenna Gaussian Channels. European Transactions on Telecommunications, Vol. 10, No. 6, pp. 585-595

Tepedelenlioglu, C.; Abdi, A. \& Giannakis, G. B. (2003). The Ricean K Factor: Estimation and Performance Analysis, IEEE Trans. Wireless Commun., Vol. 2, No. 4, pp. 799-810 


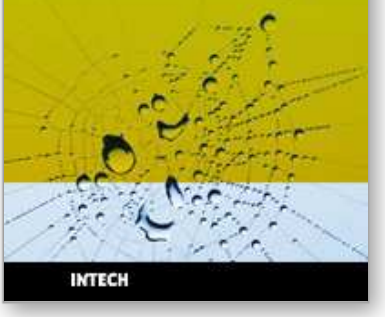

\section{Mobile and Wireless Communications Physical Layer Development and Implementatiom}

Edited by Salma Ait Fares and Fumiyuki Adachi

ISBN 978-953-307-043-8

Hard cover, 290 pages

Publisher InTech

Published online 01, January, 2010

Published in print edition January, 2010

Mobile and Wireless Communications have been one of the major revolutions of the late twentieth century. We are witnessing a very fast growth in these technologies where mobile and wireless communications have become so ubiquitous in our society and indispensable for our daily lives. The relentless demand for higher data rates with better quality of services to comply with state-of-the art applications has revolutionized the wireless communication field and led to the emergence of new technologies such as Bluetooth, WiFi, Wimax, Ultra wideband, OFDMA. Moreover, the market tendency confirms that this revolution is not ready to stop in the foreseen future. Mobile and wireless communications applications cover diverse areas including entertainment, industrialist, biomedical, medicine, safety and security, and others, which definitely are improving our daily life. Wireless communication network is a multidisciplinary field addressing different aspects raging from theoretical analysis, system architecture design, and hardware and software implementations. While different new applications are requiring higher data rates and better quality of service and prolonging the mobile battery life, new development and advanced research studies and systems and circuits designs are necessary to keep pace with the market requirements. This book covers the most advanced research and development topics in mobile and wireless communication networks. It is divided into two parts with a total of thirty-four stand-alone chapters covering various areas of wireless communications of special topics including: physical layer and network layer, access methods and scheduling, techniques and technologies, antenna and amplifier design, integrated circuit design, applications and systems. These chapters present advanced novel and cutting-edge results and development related to wireless communication offering the readers the opportunity to enrich their knowledge in specific topics as well as to explore the whole field of rapidly emerging mobile and wireless networks. We hope that this book will be useful for students, researchers and practitioners in their research studies.

\section{How to reference}

In order to correctly reference this scholarly work, feel free to copy and paste the following:

Leilei Liu, Wei Hong, Nianzu Zhang, Haiming Wang and Guangqi Yang (2010). MIMO Channel Characteristics in Line-of-Sight Environments, Mobile and Wireless Communications Physical Layer Development and Implementatiom, Salma Ait Fares and Fumiyuki Adachi (Ed.), ISBN: 978-953-307-043-8, InTech, Available from: http://www.intechopen.com/books/mobile-and-wireless-communications-physical-layer-developmentand-implementatiom/mimo-channel-characteristics-in-line-of-sight-environments

\section{INTECH}




\section{InTech Europe}

University Campus STeP Ri

Slavka Krautzeka 83/A

51000 Rijeka, Croatia

Phone: +385 (51) 770447

Fax: +385 (51) 686166

www.intechopen.com

\section{InTech China}

Unit 405, Office Block, Hotel Equatorial Shanghai

No.65, Yan An Road (West), Shanghai, 200040, China

中国上海市延安西路65号上海国际贵都大饭店办公楼 405 单元

Phone: +86-21-62489820

Fax: $+86-21-62489821$ 
(C) 2010 The Author(s). Licensee IntechOpen. This chapter is distributed under the terms of the Creative Commons Attribution-NonCommercial-ShareAlike-3.0 License, which permits use, distribution and reproduction for non-commercial purposes, provided the original is properly cited and derivative works building on this content are distributed under the same license. 